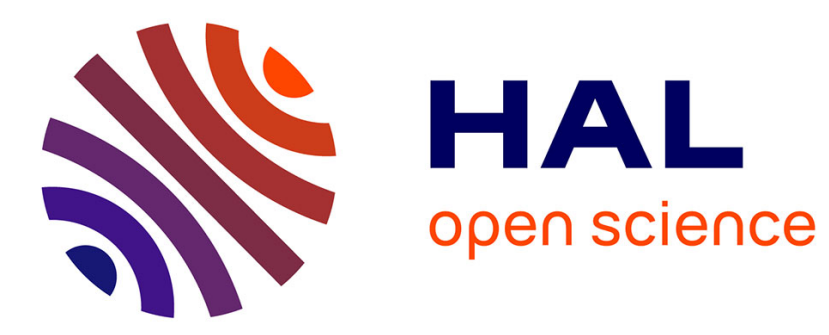

\title{
Identification for gain-scheduling: a balanced subspace approach
}

\author{
Marco Lovera, Guillaume Mercère
}

\section{To cite this version:}

Marco Lovera, Guillaume Mercère. Identification for gain-scheduling: a balanced subspace approach. American Control Conference 2007, ACC'07, Jul 2007, New York, United States. pp.CDROM. hal00164365

\section{HAL Id: hal-00164365 https://hal.science/hal-00164365}

Submitted on $20 \mathrm{Jul} 2007$

HAL is a multi-disciplinary open access archive for the deposit and dissemination of scientific research documents, whether they are published or not. The documents may come from teaching and research institutions in France or abroad, or from public or private research centers.
L'archive ouverte pluridisciplinaire HAL, est destinée au dépôt et à la diffusion de documents scientifiques de niveau recherche, publiés ou non, émanant des établissements d'enseignement et de recherche français ou étrangers, des laboratoires publics ou privés. 


\title{
Identification for gain-scheduling: a balanced subspace approach
}

\author{
Marco Lovera and Guillaume Mercère
}

\begin{abstract}
The problem of deriving MIMO parameterdependent models for gain-scheduling control design from data generated by local identification experiments is considered and a numerically sound approach is proposed, based on subspace identification ideas combined with the use of suitable properties of balanced state space realisations. Simulation examples are used to demonstrate the performance of the proposed approach.
\end{abstract}

\section{INTRODUCTION}

In the practice of control engineering there is a significant number of applications in which a single control system must be designed in order to guarantee the satisfactory closed loop operation of a given plant in many different operating conditions. The gain scheduling approach to the problem, which has been part of the engineering practice for decades, can be roughly summarised as follows: find one or more scheduling variables which can completely parameterise the operating space of interest for the system to be controlled; define a parametric family of linearised models for the plant associated with the set of operating points of interest; finally, design a parametric controller which can both ensure the desired control objectives in each operating point and an acceptable behaviour during (slow) transients between one operating condition and the other. As is well known, a wide body of design techniques is now available for this problem (see, e.g., [1]-[3]), which can be reliably solved, provided that a suitable model in parameter-dependent form has been derived.

This modelling problem, however, raises a number of significant issues. While the literature on non linear identification can now provide advanced tools for the estimation of a wide variety of model classes, in such a case it would be useful to separate conventional input variables from scheduling variables (i.e., variables defining the operating point of the plant), by letting them enter the model in distinct ways ( [4], [5]).

Linear Parametrically Varying (LPV) models have been recently proposed as a way of dealing with this kind of problems. A LPV model is linear in the parameters and a vector of scheduling variables enters the system matrices in an affine or linear fractional way ( [6]-[11]). Such a representation for general nonlinear models can be useful in view of control design using modern robust control theory (see, e.g., [12])

This paper has been supported by the Italian MIUR project Identification and Adaptive Control of Industrial Systems

M. Lovera is with the Dipartimento di Elettronica e Informazione, Politecnico di Milano, Milano, Italy. lovera@elet.polimi.it

G. Mercère is with the Laboratoire d'Automatique et d'Informatique Industrielle (LAII), Poitiers, France. guillaume.mercere@univ-poitiers.fr and gain-scheduling control techniques. Unfortunately, the underlying assumption of the above cited LPV identification techniques is that the identification procedure can rely on one global identification experiment in which both the control input and the scheduling variables are (persistently) excited in a simultaneous way. This assumption may not be a reasonable one in many applications, in which it would be desirable to try and derive a parameter-dependent model on the basis of local experiments only, i.e., experiments in which the scheduling variable is held constant and only the control input is excited.

Such a viewpoint has been considered in [13]-[15], where numerical procedures for the construction of parametric models for gain scheduling on the basis of local experiments and for the interpolation of local controllers have been proposed. The aim of this paper is to further elaborate on such approaches, in order to provide a number of extensions, namely:

- The identification of local models is performed using subspace techniques, so enabling the straightforward treatment of MIMO as well as SISO modelling problems, in state space form;

- Well known properties of subspace methods with respect to balancing of identified models are exploited in order to improve the numerical reliability of the subsequent model interpolation procedure.

The paper is organised as follows. The considered system identification problem is formulated in Section II, while the proposed approach is described in Section III. Finally, some simulation results are presented in Section IV.

\section{Problem Statement}

The system identification problem considered in this paper can be summarised as follows. Consider the MIMO linear parametrically-varying system given by

$$
\begin{aligned}
& \dot{x}=A(p) x+B(p) u \\
& y=C(p) x+D(p) u
\end{aligned}
$$

where $u \in \mathbb{R}^{m}, y \in \mathbb{R}^{l}, x \in \mathbb{R}^{n}$ and $p \in \mathbb{R}^{r}$ and assume that the results of a number of $P$ identification experiments are available, associated with the operation of the system near $P$ different values of the parameter vector $p$. The aim of the identification procedure proposed in this paper is to determine a set of parameter dependent matrices $\hat{A}(p), \hat{B}(p), \hat{C}(p)$ and $\hat{D}(p)$ either in affine (A) or linear fractional transformation (LFT) which can provide a good approximation of the system (2) over the considered range of operating points. 
III. A BALANCED SUBSPACE APPROACH TO IDENTIFICATION FOR GAIN SCHEDULING

The approach to the problem of identification for gain scheduling proposed in this paper can be summarised in the following steps:

- Linear discrete-time state space models are estimated for each operating point, using a frequency-domain SMI algorithm (see [16]);

- The identified models are balanced using the numerical algorithm of [17] (as implemented in the Matlab Control Toolbox);

- If necessary, the balanced models are converted to continuous-time using a bilinear transformation;

- Finally, the parameter-dependent model is obtained by direct interpolation of the state-space matrices of the local models, made possible by the unique properties of balanced realisations (see [17]), and can eventually be converted to LFT form using results from the LPV identification literature [6].

Each of the above mentioned steps will be described in detail in the following.

\section{A. Frequency domain subspace identification}

The problem of frequency-domain identification of MIMO linear time invariant systems using subspace methods has been extensively studied (see [16], [18]-[20]). More precisely, most of the developed methods try to fit a discrete time state space model to different types of measured frequency data, either Fourier transforms of the I/O data or samples of the frequency response of the system [16], [18] at some discrete set of frequencies. For reasons which will become clearer in the following, in this paper we focus on a particular algorithm, which was originally proposed in [16]. Such algorithm assumes that the "true" system is of finite order $n$ and can be described by a discrete-time linear state space model

$$
\begin{aligned}
x(t+1) & =A x(t)+B u(t) \\
y(t) & =C x(t)+D u(t),
\end{aligned}
$$

with $u(t) \in \mathbb{R}^{m}$ the input vector, $y(t) \in \mathbb{R}^{p}$ the output vector and $x(t) \in \mathbb{R}^{n}$ the state vector. The corresponding frequency response will be denoted as

$$
G\left(e^{j \omega}\right)=\sum_{k=0}^{\infty} g_{k} e^{-j \omega k}=C\left(e^{j \omega} I-A\right)^{-1} B+D
$$

with $g_{k}$ the Markov parameters

$$
g_{k}=\left\{\begin{array}{l}
D \text { for } k=0 \\
C A^{k-1} B \text { for } k>0
\end{array} .\right.
$$

The identification problem considered in [16] can be formulated as follows: given $M$ noise corrupted samples of the frequency response of the system

$$
G_{k}=G\left(e^{j \omega_{k}}\right)+n_{k}, k=1, \ldots, M
$$

on a set of uniformly spaced frequencies $\omega_{k}=\frac{\pi k}{M}, k=$ $1, \ldots, M$, where $n_{k}$ is a zero mean complex random variable with covariance

$$
\mathbb{E}\left\{\left[\begin{array}{l}
\Re\left\{n_{k}\right\} \\
\Im\left\{n_{k}\right\}
\end{array}\right]\left[\Re\left\{n_{s}^{T}\right\} \quad \Im\left\{n_{s}^{T}\right\}\right]\right\}=\left[\begin{array}{cc}
\frac{1}{2} R_{k} & 0 \\
0 & \frac{1}{2} R_{s}
\end{array}\right] \delta_{k s}
$$

such as this covariance function is uniformly bounded, i.e., $R_{k} \leq R$, estimate the system matrices up to a similarity transformation and, by extension, the transfer function of the process such as

$$
\lim _{M \rightarrow \infty}\|\hat{G}-G\|_{\infty}=0
$$

with probability 1 .

The algorithm proceeds by first estimating the impulse response coefficients (5) from the available frequency response samples and subsequently applying a realization algorithm. The Markov parameters are calculated by using the two sided inverse discrete Fourier transform

$$
\hat{h}_{i}=\frac{1}{2 M} \sum_{k=0}^{2 M-1} G_{k} e^{\frac{j 2 \pi i k}{2 M}}, \quad i=0, \ldots, 2 M-1
$$

with $G_{M+k}=G_{M-k}^{*}, \quad k=1, \ldots, M-1$. Knowing these estimates, the following block Hankel matrix can be built

$$
\hat{H}_{q r}=\left[\begin{array}{cccc}
\hat{h}_{1} & \hat{h}_{2} & \cdots & \hat{h}_{r} \\
\hat{h}_{2} & \hat{h}_{3} & \cdots & \hat{h}_{r+1} \\
\vdots & \vdots & \ddots & \vdots \\
\hat{h}_{q} & \hat{h}_{q+1} & \cdots & \hat{h}_{q+r-1}
\end{array}\right] \in \mathbb{R}^{q p \times r m}
$$

with $q>n, r \geq n$ and $q+r \leq 2 M$.

Since this Hankel matrix can be factored as [16]

$$
\hat{H}_{q r}=\mathcal{O}_{q}\left(I-A^{2 M}\right)^{-1} \mathcal{C}_{r}
$$

with

$$
\begin{aligned}
\mathcal{O}_{q} & =\left[\begin{array}{llll}
C^{T} & (C A)^{T} & \cdots & \left(C A^{q-1}\right)^{T}
\end{array}\right]^{T} \\
\mathcal{C}_{r} & =\left[\begin{array}{llll}
B & A B & \cdots & A^{r-1} B
\end{array}\right],
\end{aligned}
$$

computing the singular value decomposition (SVD) of $\hat{H}_{q r}$ we get

$$
\hat{H}_{q r}=\hat{U} \hat{\Sigma} \hat{V}^{T}=\left[\begin{array}{ll}
\hat{U}_{s} & \hat{U}_{n}
\end{array}\right]\left[\begin{array}{cc}
\hat{\Sigma}_{s} & 0 \\
0 & \hat{\Sigma}_{n}
\end{array}\right]\left[\begin{array}{c}
\hat{V}_{s}^{T} \\
\hat{V}_{n}^{T}
\end{array}\right],
$$

and we can estimate the observability subspace as $\hat{\mathcal{O}}_{q}=\hat{U}_{s}$ or $\hat{\mathcal{O}}_{q}=\hat{U}_{s} \hat{\Sigma}_{s}^{1 / 2}$. Finally, the extraction of the system matrices can be carried out using conventional subspace techniques.

\section{B. Balancing of the identified models}

Once $P$ local models have been obtained, the problem of recovering the whole parameter-dependent system has to be faced. It turns out that this task is greatly simplified if the local models are converted to a suitable balanced form. In order to clarify this point, the main properties of internally balanced realisations have to be briefly summarised ( [21], [22]). 
Definition 3.1: Consider the discrete-time system (3) and the matrices

$$
\mathcal{W}_{o}=\sum_{k=0}^{\infty}\left(A^{k}\right)^{T} C^{T} C A^{k}, \quad \mathcal{W}_{c}=\sum_{k=0}^{\infty} A^{k} B B^{T}\left(A^{k}\right)^{T}
$$

which are respectively known as the observability and controllability Gramians of the state space system (3). The state space realization is internally balanced if

$$
\mathcal{W}_{o}=\mathcal{W}_{c}=\Sigma=\operatorname{diag}\left(\sigma_{1} \cdots \sigma_{n}\right),
$$

where $\left\{\sigma_{i}\right\}_{i=1}^{n}$ are the singular values of $\mathcal{W}_{c} \mathcal{W}_{o}$.

Definition 3.2: Consider the observability matrix $\mathcal{O}_{q}$ and the controllability matrix $\mathcal{C}_{r}$ defined in (12) and (13). The corresponding state space realization is $q r$ internally balanced if $\mathcal{O}_{q}^{T} \mathcal{O}_{q}=\mathcal{C}_{r} \mathcal{C}_{r}^{T}=\Sigma$.

In the noise free case, the frequency domain realization algorithm presented in the previous subsection leads to the following factorization [16]

$$
\hat{H}_{q r}=\mathcal{O}_{q}\left(I-A^{2 M}\right)^{-1} \mathcal{C}_{r}
$$

with $q>n, r \geq n$ and $q+r \leq 2 M$. Now, the SVD (14) allows to write

$$
\hat{H}_{q r}=\hat{U}_{s} \hat{\Sigma}_{s}^{1 / 2} \Sigma_{s}^{T / 2} \hat{U}_{s}^{T}
$$

Thus, the estimates of the observability and controllability matrices satisfy

$$
\hat{\mathcal{O}}_{q}=\hat{U}_{s} \hat{\Sigma}_{s}^{1 / 2}, \quad \hat{\mathcal{C}}_{r}=\Sigma_{s}^{T / 2} \hat{U}_{s}^{T}
$$

Hence, we obtain

$$
\begin{aligned}
\mathcal{O}_{q}^{T} \mathcal{O}_{q} & =\hat{\Sigma}_{s} \\
\mathcal{C}_{r} \mathcal{C}_{r}^{T} & =\left(I-A^{2 M}\right) \hat{\Sigma}_{s}\left(I-A^{2 M}\right)^{T} .
\end{aligned}
$$

For a stable system, the state space realization given by the proposed algorithm is therefore $q r$ balanced when $M$ tends to infinity.

In order to improve the numerical performance of the algorithm, it is proposed to fix the state space basis of the estimated matrices by finding a similarity transformation $T$ such that $\left\{T^{-1} \hat{A} T, T^{-1} \hat{B}, \hat{C} T, \hat{D}\right\}$ is in internally balanced form. To this purpose, we use the algorithm first derived in [17], which consists in the following steps:

1) Compute the Gramians $\mathcal{W}_{o}$ and $\mathcal{W}_{c}$ of the identified model;

2) apply a Cholesky factorisation to obtain $\mathcal{W}_{o}=L_{o} L_{o}^{T}$, $\mathcal{W}_{c}=L_{c} L_{c}^{T}$, where $L_{o}$ and $L_{c}$ are the lower triangular factors of the Gramians;

3) compute the SVD $L_{o}^{T} L_{c}=U \Sigma V^{T}$.

The balancing transformation is then defined by $T=$ $L_{c} V \Sigma^{-1 / 2}, T^{-1}=\Sigma^{-1 / 2} U^{T} L_{o}^{T}$.

The most interesting properties of balanced realisations, as far as this contribution is concerned, is associated with the uniqueness properties of the balancing transformation (see [17] and [21] for details). Essentially, the question turns on the eigenvalues (real and nonnegative) of the product of the reachability and observability Gramians, and their corresponding eigenvectors, i.e., the columns of the balancing transformation $T$. If these eigenvalues are distinct, then the corresponding eigenvectors are uniquely determined to within sign, i.e., $T$ is essentially unique. If, on the other hand, two or more eigenvalues are repeated, then their corresponding eigenvectors can be rotated arbitrarily in the corresponding eigenspace. This, in turn, implies that as long as the eigenvalues are distinct, if the true system exhibits a smooth dependence from the scheduling parameter $p$, so that the overall parameter dependent model can be directly reconstructed from the identified local models.

Since, in general, the assumption of distinct eigenvalues cannot be guaranteed a priori, as far as our identification problem is concerned, the following situations may arise:

- If the elements of the state space matrices of the identified local models exhibit a smooth variation as a function of the parameter $p$, then the overall parameterdependent model can be directly recovered using the interpolation techniques outlined in the following subsections.

- If, instead, the behaviour of the elements of the state space matrices exhibit abrupt sign changes (associated with sign changes in the columns of the balancing transformations), then one should check for situations in which the eigenvalues of the balanced Gramians are not distinct.

- If signs changes are present but the eigenvalues are distinct, then a "mild" nonuniqueness is occurring, which can simply be corrected by adjusting the signs of the "stray" matrix elements;

- If, on the other hand, sign changes are associated with non unique eigenvalues, then the best solution is to momentarily neglect the local model giving rise to the problem and performing the model interpolation (as described in the following subsections) using $P-1$ local models only.

\section{From discrete-time to continuous-time}

Until now, only discrete time frequency domain identification has been considered. If the goal of the identification procedure, however, is to work out a continuous-time model for the system, then the well known bilinear transformation can be applied. In particular, explicit formulas exist for the direct transformation of the state space matrices from discrete to continuous time (see [16]):

$$
\begin{aligned}
& A_{c}=\frac{2}{T_{s}}(I+A)^{-1}(A+I), \quad B_{c}=\frac{2}{\sqrt{T_{s}}}(I+A)^{-1} B \\
& C_{c}=\frac{2}{\sqrt{T_{s}}} C(I+A)^{-1}, \quad D_{c}=D-C(I+A)^{-1} B,
\end{aligned}
$$

where $T_{s}$ is the sampling period. The main advantage of the bilinear transformation with respect to other approaches to the discrete/continous conversion is that this transformation preserves balancing, i.e., when applied to a discrete-time system in balanced form, yields a balanced continuous-time system (see also [23]). Therefore, the above comments on the 
essential uniqueness of balanced realisations in the discretetime can be successfully exploited for model interpolation also in the continuous-time case.

\section{Model interpolation}

Once the elements of the state space matrices of the system have been estimated following the above steps, a number of options are available as far as the derivation of the actual parameter dependent model is concerned. The first, and simplest, would be to directly fit to the system matrices of the local models using suitable regressors formed from the scheduling parameter $p$. This would directly yield a parameter dependent model in so-called affine form (LPVA), i.e.,

$$
\begin{aligned}
& \dot{x}=A(\delta) x+B(\delta) u \\
& y=C(\delta) x+D(\delta) u
\end{aligned}
$$

where $\delta \in \mathbb{R}^{d}$ is the vector of regressors (formed from linear or non linear functions of the elements of $p$ ) such that the parameter dependent matrices can be written as

$$
A(\delta)=A_{0}+A_{1} \delta_{1}+\ldots+A_{d} \delta_{s}
$$

and similarly for $B(\delta), C(\delta)$ and $D(\delta)$. In some applications, however, it is desirable to obtain as output of the system identification procedure a model in so-called LPV-LFT form, i.e., in which the state space matrices are expressed as a linear fractional transformation over a suitable linear operator representing time-varying parameters:

$$
\begin{gathered}
\dot{x}=A x+B_{0} w+B_{1} u \\
z=C_{0} x+D_{00} w+D_{01} u \\
y=C_{1} x+D_{10} w+D_{11} u \\
w=\Delta z, \quad \Delta=\operatorname{diag}\left(\delta_{1} I_{r_{1}} \ldots \delta_{d} I_{r_{d}}\right)
\end{gathered}
$$

and $w, z \in \mathbb{R}^{r}, r=r_{1}+\ldots+r_{d}$.

As is well known in the robust control literature and also pointed out in [6], [8], LPV-A and LPV-LFT representations are related to each other. Denoting the composition of the system matrices

$$
M(\delta)=\left[\begin{array}{ll}
A(\delta) & B(\delta) \\
C(\delta) & D(\delta)
\end{array}\right]=M_{0}+M_{1} \delta_{1}+\ldots+M_{d} \delta_{d},
$$

by expressing each of the $M_{i}^{\prime} s, i=1, \ldots, d$ by means of a rank $r_{i}$ decomposition as $M_{i}=U_{i} V_{i}$ one can write $M(\delta)$ as $M(\delta)=M_{0}+U \Delta V$, where $U=\left[U_{1} \ldots U_{d}\right]$, $V=\left[V_{1}^{T} \ldots V_{d}^{T}\right]^{T}$ and $\Delta$ is given by equation (27).

The obtained form for the system matrices coincides with the one which is obtained in the special case of a linear fractional transformation characterised by having $D_{00}=0$, hence the transformation between the two forms.

\section{E. Comments and discussion}

As mentioned in the Introduction, the proposed approach seems to offer a useful trade-off between the two classes of solutions to this problem which have been so far proposed in the literature. On one hand, subspace methods for the identification of LPV state space models such as the ones in [10] provide a very general way of dealing with the problem, at the cost of critical requirements on the experimental conditions which might not be easily realisable in many applications. On the other hand, the technique of [13], [14] has the advantage of being much closer to the actual practice of system identification but suffers from the numerical drawbacks associated with the adoption of a fixed (generally not well conditioned) basis for the manipulation of state space models.

\section{Simulation EXAMPLES}

\section{Example 1}

Consider the parameter-dependent system given by the equations

$$
\begin{aligned}
x(k+1) & =A(p) x(k)+B u(k) \\
y(k) & =C x(k)
\end{aligned}
$$

where

$$
\begin{gathered}
A=\left[\begin{array}{ccc}
0 & 1 & 0 \\
0 & 0 & 1 \\
0.01 p & -(p-0.004) & -0.39
\end{array}\right] \\
B=\left[\begin{array}{lll}
0 & 0 & 1
\end{array}\right]^{T}, \quad C=\left[\begin{array}{lll}
0.02 & 0 & -0.5
\end{array}\right]
\end{gathered}
$$

and the parameter $p$ ranges in the interval from 0.1 to 0.95 . The Bode plots of the frequency responses associated with the system for different values of $p$ are given in Figure 1. As can be seen, the dynamics of the system is significantly affected by the parameter variations. For this system it is

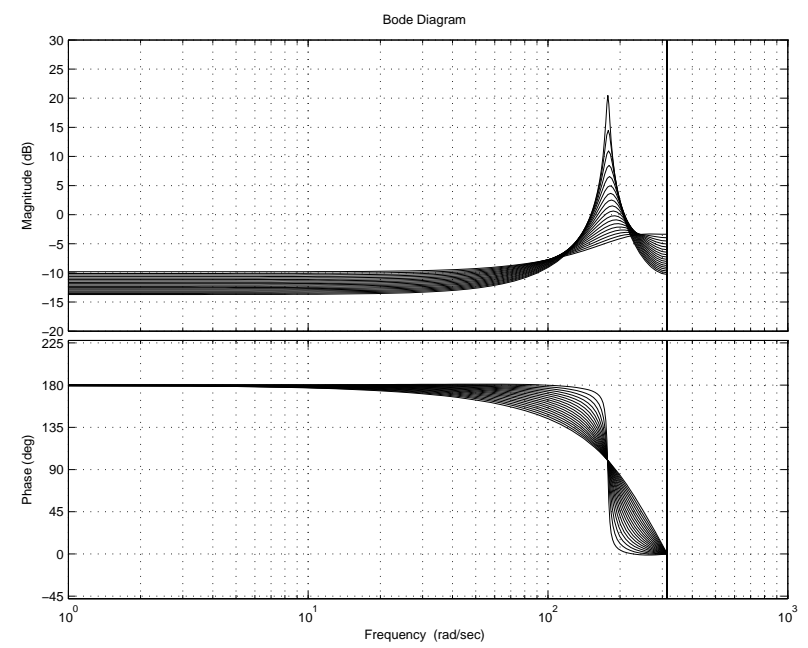

Fig. 1: Example 1: frequency response of the parameter dependent system for $p=(0.1: 0.05: 0.95)$.

assumed that frequency domain data, i.e., measurements of its frequency response around each operating point are available. On the basis of such data, linear time-invariant state space models have been identified and balanced using the SMI algorithm outlined in Section III. The resulting model parameters (elements of the estimated $A, B, C$ and $D$ matrices) are presented in Figure 2. Its is apparent from the Figure that, as expected from the previous theoretical 


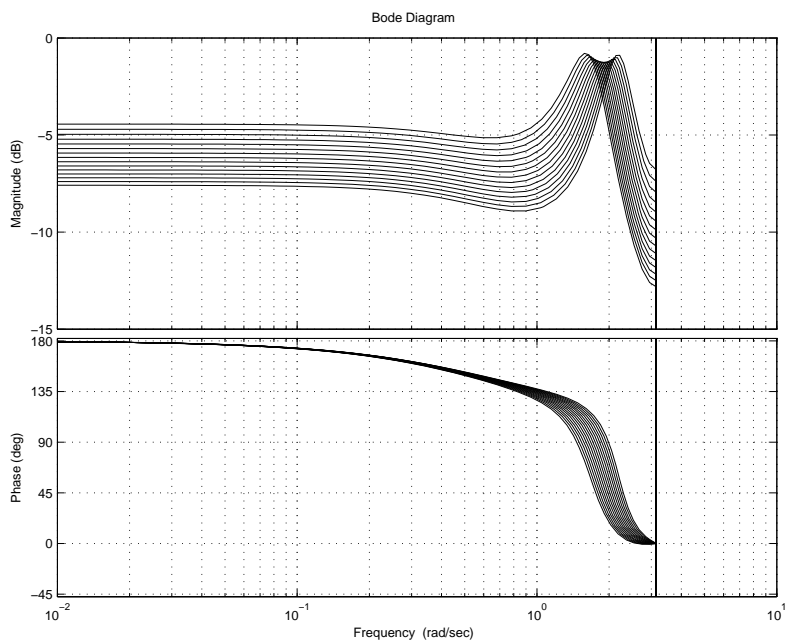

Fig. 5: Example 2: frequency response of the parameter dependent system for $p=(0.1: 0.05: 0.8)$.
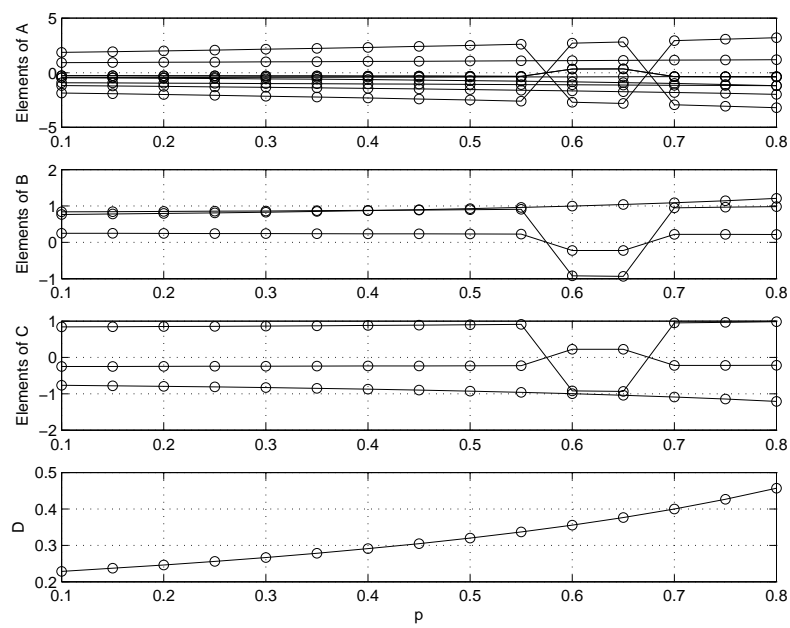

Fig. 6: Example 2: elements of the estimated $A, B, C$ and $D$ matrices as functions of $p$.

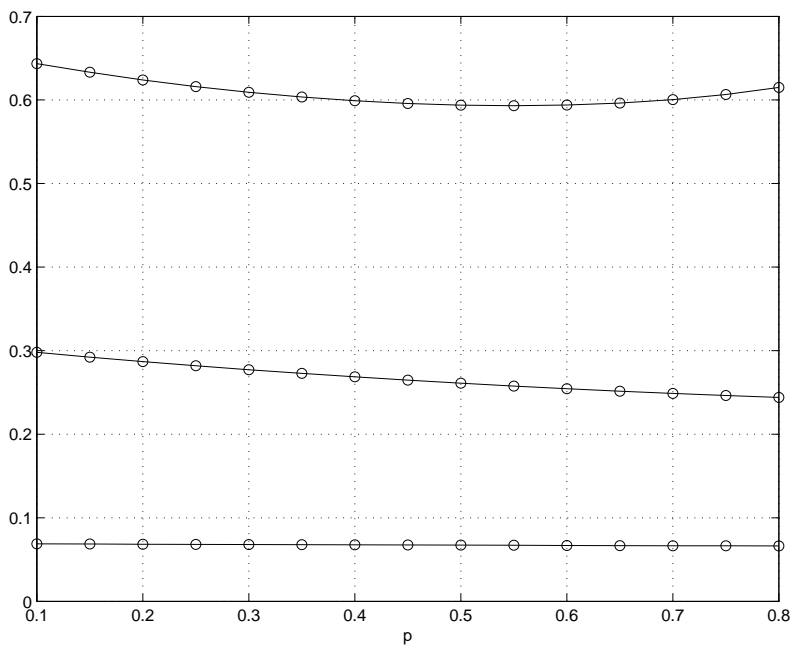

Fig. 7: Example 2: eigenvalues of the balanced Gramian matrices, as function of $p$.

\section{REFERENCES}

[1] P. Apkarian and R. J. Adams, "Advanced Gain-Scheduling Techniques for Uncertain Systems," IEEE Transactions on Control System Technology, vol. 6, pp. 21-32, 1998.

[2] I. Kaminer, A. M. Rascoal, P. P. Karghonekar, and E. E. Coleman, "A Velocity Algorithm for the Implementation of Gain-Scheduled Controllers," Automatica, vol. 31, pp. 1185-1191, 1995.

[3] J. S. Shamma and M. Athans, "Analysis of Gain-Scheduled Control for Nonlinear Plants," IEEE Transactions on Automatic Control, vol. 35, pp. 898-907, 1990.

[4] A. Skeppstedt, L. Ljung, and M. Millnert, "Construction of composite models from observed data," International Journal of Control, vol. 55, pp. 141-152, 1992.

[5] T. A. Johansen and B. A. Foss, "Identification of Non-Linear System Structure and Parameters using Regime Decomposition," Automatica, vol. 31, pp. 321-326, 1995.

[6] L. H. Lee and K. Poolla, "Identification of Linear Parameter-Varying Systems via LFT," in Proc. of the 35th IEEE Conference on Decision and Control, Japan, 1996, pp. 1545-1550.

[7] M. Nemani, R. Ravikanth, and B. Bamieh, "Identification of Linear Parameter-Varying Systems," in Proc. of the 34th IEEE Conference on Decision and Control, 1995.

[8] M. Lovera, M. Verhaegen, and C. T. Chou, "State space identification of MIMO linear parameter varying models," in Proc. of the International Conference on Mathematical Theory of Networks and Systems, Padova, Italy, 1996.

[9] B. Bamieh and L. Giarré, "Identification of linear parameter varying models," in Proc. of the IEEE Conference on Decision and Control, Phoenix, AZ, USA, 1999.

[10] V. Verdult, "Nonlinear system identification: a state space approach," $\mathrm{Ph} . \mathrm{D}$. dissertation, University of Twente, 2002.

[11] F. Previdi and M. Lovera, "Identification of a class of nonlinear parametrically varying models," International Journal on Adaptive Control and Signal Processing, vol. 17, pp. 33-50, 2003.

[12] P. Apkarian and P. Gahinet, "A Convex Characterization of GainScheduling $H_{\infty}$ Controllers," IEEE Transactions on Automatic Control, vol. 40, pp. 853-864, 1995.

[13] M. Steinbuch, R. van de Molengraft, and A. van der Voort, "Experimental modelling and LPV control of a motion system," in Proceedings of the 2003 American Control Conference, Denver, USA, 2003.

[14] J. van Helvoort, M. Steinbuch, P. Lambrechts, and R. van de Molengraft, "Analytical and experimental modelling for gain-scheduling of a double scara robot," in Proceedings of the 3rd IFAC Symposium on Mechatronic Systems, Sydney, Australia, 2004.

[15] B. Paijmans, W. Symens, H. V. Brussel, and J. Swevers, "A gainscheduling-control technique for mechatronic systems with positiondependent dynamics," in Proceedings of the 2006 American Control Conference, Minneapolis, USA, 2006

[16] T. McKelvey, H. Akçay, and L. Ljung, "Subspace based multivariable system identification from frequency response data," IEEE Transactions on Automatic Control, vol. 41, pp. 960-978, 1996.

[17] A. Laub, M. Heath, C. Paige, and R. Ward, "Computation of system balancing transformations and other applications of simultaneous diagonalization algorithms," IEEE Transactions on Automatic Control, vol. 32, pp. 115-122, 1987.

[18] R. Jacques, K. Liu, and D. Miller, "Identification of highly accurate low order state space models in the frequency domain," Signal Processing, vol. 52, pp. 195-207, 1996.

[19] P. Van Overschee and B. De Moor, "Continuous time frequency domain subspace system identification," Signal Processing, vol. 52, pp. 179-194, 1996

[20] R. Pintelon, "Frequency domain subspace system identification using non parametric noise models," Automatica, vol. 38, pp. 1295-1311, 2002.

[21] B. Moore, "Principal component analysis in linear systems: controllability, observability and model reduction," IEEE Transactions on Automatic Control, vol. 26, pp. 17-32, 1981.

[22] M. Moonen and J. Ramos, "A subspace algorithm for balanced state space system identification," IEEE Transactions on Automatic Control, vol. 38, pp. 1727-1729, 1993

[23] U. Al-Saggaf and G. Franklin, "Model reduction via balanced realizations: an extension and frequency weighting techniques," IEEE Transactions on Automatic Control, vol. 33, pp. 687-692, 1988. 